\title{
BMJ Open Longitudinal impact of interprofessional education on attitudes, skills and career trajectories: a protocol for a quasi- experimental study in New Zealand
}

\author{
Ben Darlow, ${ }^{1}$ Melanie Brown, ${ }^{1}$ Peter Gallagher, ${ }^{2}$ Lesley Gray, ${ }^{1}$ Eileen McKinlay, ${ }^{1}$ \\ Gordon Purdie, ${ }^{3}$ Christine Wilson, ${ }^{1}$ Sue Pullon, ${ }^{1}$ on behalf of LIP Study Group
}

To cite: Darlow B, Brown M, Gallagher P, et al. Longitudinal impact of interprofessional education on attitudes, skills and career trajectories: a protocol for a quasiexperimental study in New Zealand. BMJ Open 2018;8:e018510. doi:10.1136/ bmjopen-2017-018510

- Prepublication history for this paper is available online. To view these files, please visit the journal online (http://dx.doi. org/10.1136/bmjopen-2017018510).

Received 4 July 2017 Revised 28 October 2017 Accepted 16 November 2017

\section{Check for updates}

1Department of Primary Health Care and General Practice, University of Otago, Wellington, New Zealand

${ }^{2}$ Education Unit, University of Otago, Wellington, New Zealand ${ }^{3}$ Biostatistical Group, Dean's Department, University of Otago, Wellington, New Zealand

Correspondence to

Dr Ben Darlow;

ben.darlow@otago.ac.nz

\section{ABSTRACT}

Introduction Interprofessional practice is recognised as an important element of safe and effective healthcare. However, few studies exist that evaluate how preregistration education contributes to interprofessional competencies, and how these competencies develop throughout the early years of a health professional's career. This quasiexperimental study will gather longitudinal data during students' last year of preregistration training and their first 3 years of professional practice to evaluate the ongoing development of interprofessional competencies and the influence that preregistration education including an explicit interprofessional education (IPE) programme may have on these.

Methods and analysis Participants are students and graduates from the disciplines of dentistry, dietetics, medicine, nursing, occupational therapy, oral health, pharmacy and physiotherapy recruited before their final year of study. A subset of these students attended a 5-week IPE immersion programme during their final year of training. All data will be collected via five written or electronic surveys completed at 12-month intervals. Each survey will contain the Attitudes Towards Health Care Teams Scale and the Team Skills Scale, as well as quantitative and free-text items to explore vocational satisfaction, career trajectories and influences on these. Students who attend the IPE programme will complete additional free-text items to explore the effects of this programme on their careers. Quantitative analysis will compare scores at each time point, adjusted for baseline scores, for graduates who did and did not participate in the IPE programme. Associations between satisfaction data and discipline, professional setting, location and IPE participation will also be examined. Template analysis will explore freetext themes related to influences on career choices including participation in preregistration IPE.

Ethics and dissemination This study has received approval from the University of Otago Ethics Committee (D13/019). Results will be disseminated through peer-reviewed publications, conferences and stakeholder reports. Findings will inform future IPE developments and health workforce planning.

\section{INTRODUCTION}

Interprofessional practice is a collaborative model of healthcare that optimises the
Strengths and limitations of this study

- This prospective longitudinal study will explore the impact of a preregistration interprofessional education (IPE) immersion programme on long-term outcomes in a large cohort of graduates from eight health professions.

- The quasiexperimental study design will allow comparisons to be made between students who did and did not participate in an IPE immersion programme with regard to attitudes to interprofessional teams and self-assessed ability to function within a team, workplace location and vocation, and career satisfaction

- The results will improve understanding of the longterm effects of preregistration IPE.

- Allocation to the IPE intervention was non-random, and there are limitations associated with current instruments available to measure IPE outcomes. In addition, there may be insufficient power for some planned analyses.

- Although exposure to the IPE programme will be the key difference between graduates who did and did not participate, there may be other factors which influence graduates' attitudes and career choices, and these may confound analyses.

use of multiple professional skills sets to provide well-coordinated, safe, high-quality patient care. ${ }^{2}$ Interprofessional practice is particularly important in the context of populations with increasing prevalence of long-term conditions and multimorbidity. These complex health needs will be best met by the coordinated and collaborative involvement of a team of health professionals. ${ }^{34}$ High-quality interprofessional practice also reduces error, improves safety through better communication, increases collegial respect and trust, breaks down professional silos and hierarchies and improves vocational satisfaction, recruitment and retention. ${ }^{5-7}$ 
Interprofessional education (IPE) occurs when health professionals from more than one discipline intentionally learn with, from and about each other ${ }^{8}$ and is proposed as a way of improving collaborative practice. ${ }^{3}$ IPE appears to be generally well received by preregistration students (students enrolled in a programme that prepares them for professional registration or licensure), and short-term evaluations have found increases in knowledge and skills required for collaborative practice, improved student attitudes towards collaboration and also improved clinical behaviour and patient care. ${ }^{19-13}$ There is little evidence related to the maintenance of changes over time ${ }^{1}$ or the impact of IPE on career trajectories, professional behaviour or patient outcomes. ${ }^{1415}$

Few data are available to indicate how interprofessional attitudes and teamwork abilities are acquired and change over time, irrespective of exposure to IPE. ${ }^{9}$ Longitudinal studies of preregistration students have shown small or negative impacts of IPE on students' attitudes, ${ }^{16-18}$ but to our knowledge, only one study has explored changes over the transition from preregistration to postregistration. ${ }^{19}$ Pollard and Miers ${ }^{19}$ followed health professional students during their training and for 12 months postregistration. Their study found that attitudes to interprofessional practice improved during the first year of professional practice and that confidence related to communication skills and attitudes towards interprofessional relationships increased to a greater degree in those who had participated in preregistration IPE than those who had not. ${ }^{19}$ The 'Linköping IPE model' (an integrated programme of study culminating in clinical experience in an interprofessional student-run ward) has shown significant differences in interprofessional collaborative practice ability between doctors from Linköping compared with other Swedish medical schools, with Linköping graduates consistently better at working with people in other health professions. ${ }^{20}$

\section{Context of the study}

The provision of healthcare close to communities where people live is central to the New Zealand Health Strategy, ${ }^{21}$ but there is a shortage of health professionals working in rural areas and within primary healthcare. Few data exist which explore the career trajectories and choices of recently registered health professionals and the influence that preregistration education components may have on these outcomes.

The Tairāwhiti Interprofessional Education (TIPE) programme aims to provide a clinically based IPE programme which fosters interprofessional collaborative practice, enhances hauora Māori (indigenous Māori health), implements principles of long-term condition management and encourages graduates to work in rural and primary healthcare settings in New Zealand. ${ }^{22} 23$ Tairāwhiti is the name of the relatively remote region on the East Cape of the North Island of New Zealand where the programme is based. The Tairāwhiti region is economically disadvantaged and also has the highest proportion (49\%) of Māori of any area of New Zealand. The programme involves approximately 75 final-year preregistration students from the disciplines of dentistry, dietetics, medicine, nursing, occupational therapy, oral health, pharmacy and physiotherapy each year. TIPE programme staff are working clinicians from these disciplines who act as teachers and mentors. These students spend 5 weeks living in shared accommodation in the regional city of Gisborne (population approximately 30000 ) or the local town of Wairoa (population approximately 4200). ${ }^{24}$ The programme integrates elements of discipline-specific learning as well as interprofessional, rural, hauora Māori and long-term condition management learning activities and placements. Teaching and learning are provided across diverse town and rural community health settings.

\section{Study aims}

The primary aim of this study is to explore changes in (1) attitudes to interprofessional teams and teamwork abilities, (2) career intentions and choices related to professional setting and geographical location and (3) vocational satisfaction in participants who did and did not attend the TIPE programme. These factors will be observed over the final year of preregistration training and the first 3 years of professional practice in health professionals from the disciplines of dentistry, dietetics, nursing, medicine, occupational therapy, oral health, pharmacy and physiotherapy.

The secondary aim of this study is to explore interprofessional roles and experience for students who participated in the TIPE programme and the long-term influence of the TIPE programme on their ability to work interprofessionally and their career choices.

\section{METHODS AND ANALYSIS \\ Study design}

This is a longitudinal, quasiexperimental (non-equivalent groups) study of students from eight healthcare disciplines who have and have not participated in the TIPE programme. The study has five annual data collection points.

\section{Participants}

Cohort 1 participants were recruited in October 2014 (students from all disciplines except pharmacy) and February 2015 (pharmacy students). These participants were at the end of their penultimate year of preregistration training (students from the disciplines of dentistry, dietetics, medicine, nursing, occupational therapy and physiotherapy) or the start of their final year (students from the discipline of pharmacy). This included all students from a single year cohort from the disciplines of dentistry, dietetics, pharmacy, physiotherapy at the University of Otago, medicine at the University of Otago Wellington (a secondary campus of Otago University), nursing at 
the Eastern Institute of Technology and occupational therapy at Otago Polytechnic; there were no oral health students in cohort 1 . These disciplinary cohorts represented all students who were eligible to attend the TIPE programme; a subset of these students participated in the 2015 TIPE programme. A small number of students who did not attend the TIPE programme may have been exposed to less intensive and/or informal IPE opportunities, but these were unlikely to have caused contamination because these were of very short duration and low intensity in comparison with the TIPE programme. Cohort 1 participant recruitment and progress through the study is illustrated in figure 1.

Cohort 2 participants were recruited in February 2016 at the start of their final year of training. These students were from the disciplines of dentistry, dietetics, medicine, nursing, occupational therapy, oral health (not included in cohort 1 as joined the programme after this cohort was recruited), pharmacy and physiotherapy who were expected to attend the TIPE programme in 2016. Cohort

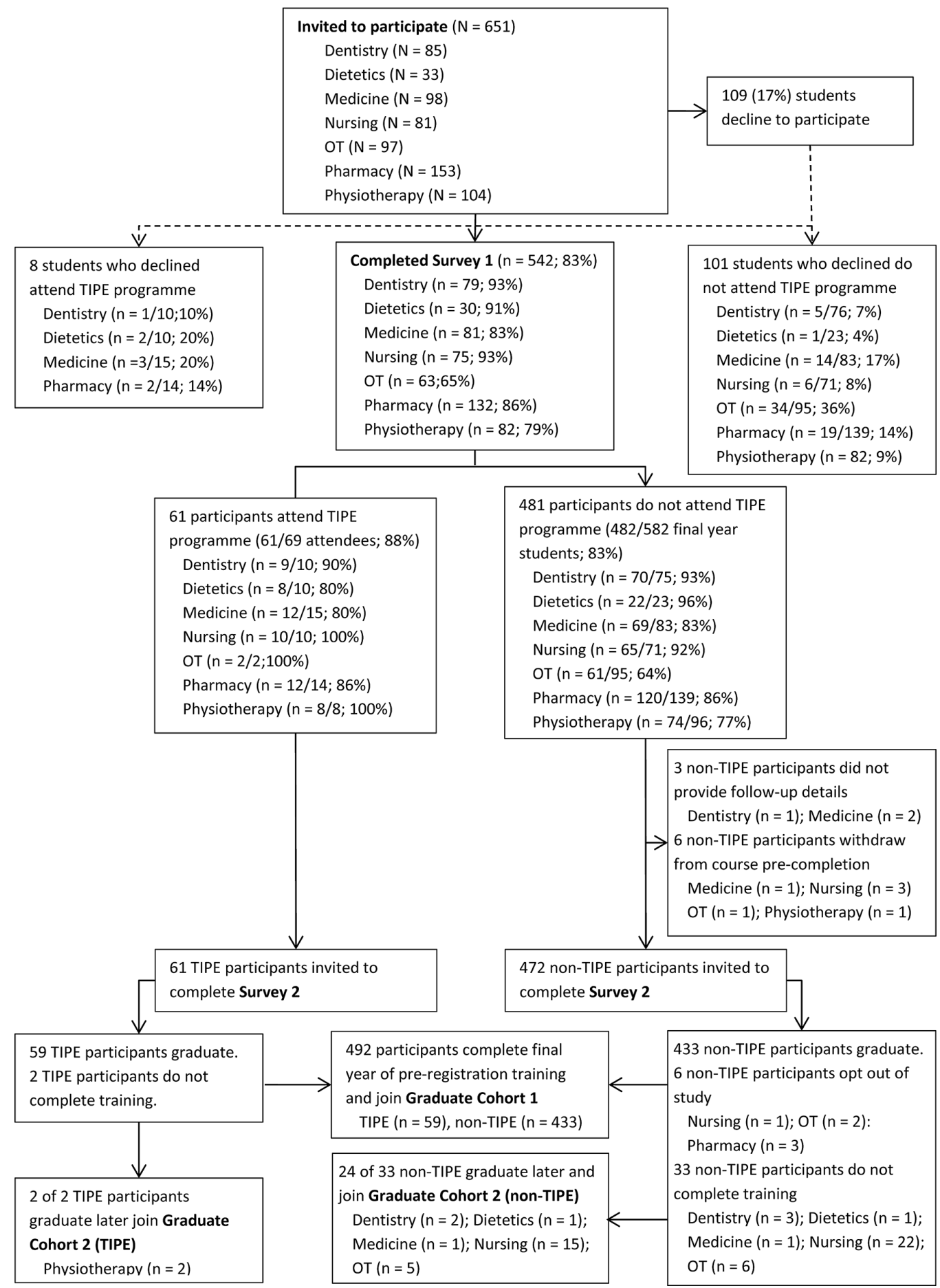

Figure 1 Participant flow and data collection for cohort 1. OT, occupational therapy; TIPE, Tairāwhiti Interprofessional Education Programme. 


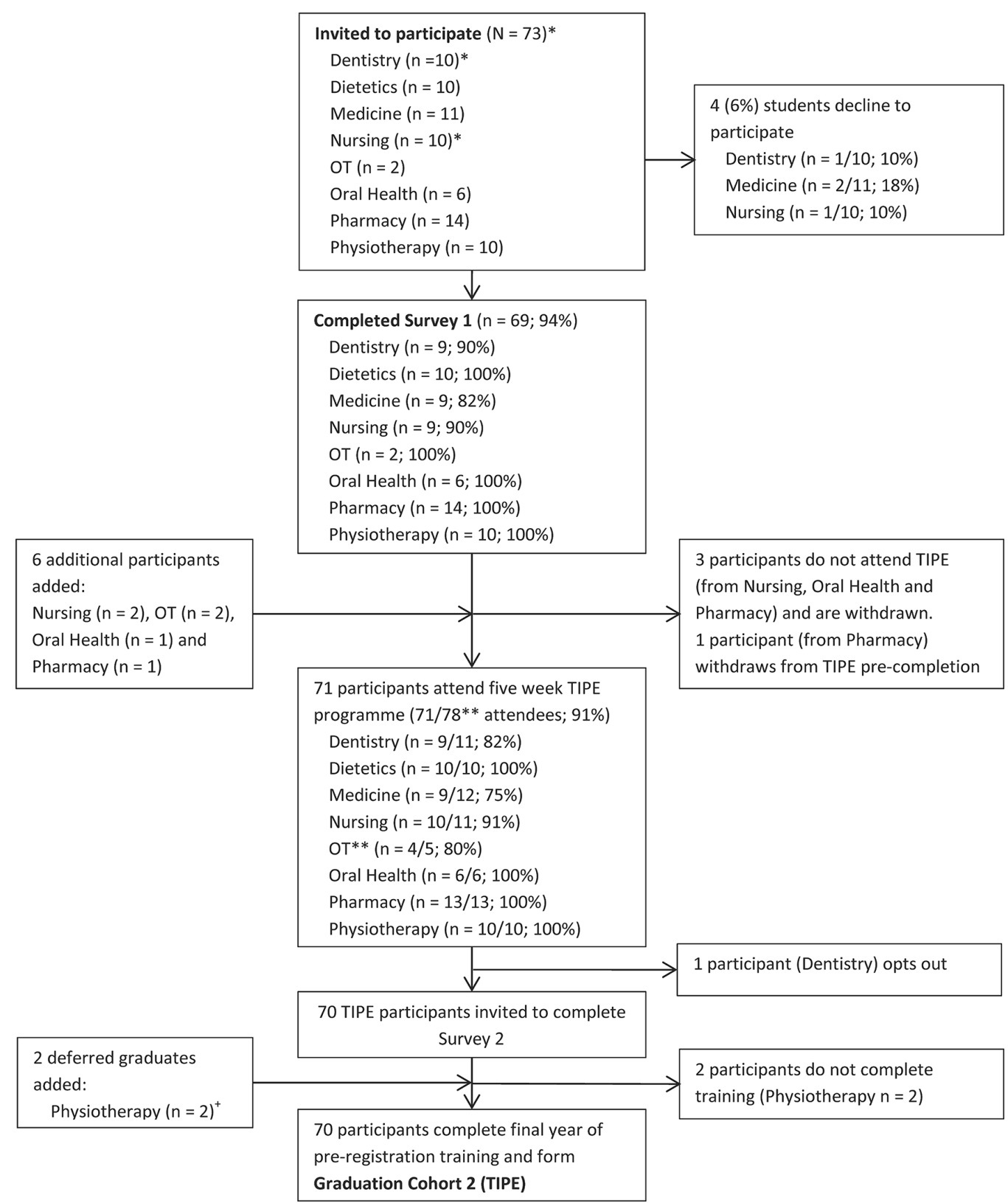

Figure 2 Participant flow and data collection for TIPE cohort 2. ${ }^{*}$ Two TIPE attendees (one from nursing and one from dentistry) attended TIPE having initially joined cohort 1 in 2014; these are not included in figure 1. ${ }^{* *}$ One additional TIPE attendee (from OT) did not join the study. +Two participants (physiotherapy=2) attended TIPE in 2015 having joined cohort 1 in 2014; they are part of the 2015 cohort 1 TIPE group for the year 1 analysis and part of the cohort 2 TIPE group for subsequent analyses. OT, occupational therapy; TIPE, Tairāwhiti Interprofessional Education Programme.

2 participant recruitment and progress through the study is illustrated in figure 2.

\section{Measures}

Demographic characteristics

Demographic characteristics collected at baseline were: gender, age, discipline, self-defined ethnicity and the type of location in which the participant had lived the longest before starting health training (major urban city (>100000 people), regional city (25000 to 100000 people), small town (5000 to 25000 people) and very small town $/ \mathrm{rural} / \mathrm{remote}(<5000))$.
Attitudes to interprofessional teams and teamwork skills Attitudes Towards Health Care Teams Scale

The participants' attitudes to interprofessional teams are assessed with the Attitudes Towards Health Care Teams Scale (ATHCTS) ${ }^{25}$ as modified by Curran et al..$^{26}$ This scale has 14 items rated on a five-point Likert scale from 'strongly disagree' (1) to 'strongly agree' (5) (with three negatively worded items that are reverse-scored). Higher scores represent more positive attitudes towards teamwork. The modified ATHCTS has been found to have high internal consistency when completed by health professional students. ${ }^{27} 28$ Three underlying constructs have 
been identified: 'quality of care delivery', 'patient-centred care' and 'team efficiency'. ${ }^{27}$

\section{Team Skills Scale}

The participants' self-assessed ability to function within an interprofessional team is assessed with the Team Skills Scale (TSS).${ }^{29}$ This scale has 17 items rated on a five-point Likert scale from 'poor' (1) to 'excellent' (5). Higher scores represent a higher self-reported skill level. The TSS has been found to have excellent internal consistency when completed by student and graduate health professionals. ${ }^{30} 31$

\section{Professional setting}

Participants' intentions with regard to the postregistration professional setting (primary healthcare/community, hospital, education (study), teaching, research, do not plan to work in healthcare, unsure, no preference and other) and type of location (major urban city ( $>100000$ people), regional city (25000 to 100000 people), small town (5000 to 25000 people), very small town/rural/ remote $(<5000)$, unsure, no preference and other) are collected through survey 2. Surveys 3 to 5 collect data regarding the participants' actual professional setting and type of location. In all surveys, participants are asked to explain why they have chosen these options using a free-text response box.

\section{Vocational satisfaction}

Satisfaction data are collected in surveys 3 to 5 through two items measured on a five-point Likert scale adapted from previous instruments. ${ }^{32-34}$ Current job or role satisfaction is assessed with the question "Taking everything into consideration, how do you feel about your current job or role?" Career satisfaction is assessed with the question "Thinking very generally, how do you feel about your overall career?"

Interprofessional practice and influence of preregistration training Additional data are collected from participants who attended the TIPE programme through free-text items developed for this study included in surveys 3 to 5 . These explore: (1) whether participants work within an interprofessional team and if so, its function, members and their role, (2) participants' experience of working or collaborating with people from different disciplines or health professions, (3) aspects of participants' preregistration education and training which they consider prepared them to work as part of an interprofessional healthcare team and (4) whether the TIPE programme had influenced participants' career choices. The fourth item was intentionally placed after the other items so that the TIPE programme is not specifically referenced until the end of the survey to minimise potential influence on responses to the other items.

\section{Data collection}

Data will be collected at five key time points (table 1):
Table 1 Data collection instruments, components and timing in relation to participants' training

\begin{tabular}{|c|c|c|}
\hline Survey & Components & Stage \\
\hline Survey 1 & $\begin{array}{l}\text { ATHCTS } \\
\text { TSS } \\
\text { Demographic items }\end{array}$ & $\begin{array}{l}\text { Prefinal year of } \\
\text { training and prior } \\
\text { to TIPE or control } \\
\text { exposure }\end{array}$ \\
\hline Survey 2 & $\begin{array}{l}\text { ATHCTS } \\
\text { TSS } \\
\text { Clinical practice intention } \\
\text { items }\end{array}$ & $\begin{array}{l}\text { Postfinal year of } \\
\text { training and after } \\
\text { TIPE or control } \\
\text { exposure }\end{array}$ \\
\hline Survey 3 & $\begin{array}{l}\text { ATHCTS } \\
\text { TSS } \\
\text { Clinical practice } \\
\text { characteristics and } \\
\text { satisfaction items } \\
\text { Free-text } \\
\text { interprofessional practice } \\
\text { items* }\end{array}$ & $\begin{array}{l}\text { One year } \\
\text { postgraduation }\end{array}$ \\
\hline Survey 4 & $\begin{array}{l}\text { ATHCTS } \\
\text { TSS } \\
\text { Clinical practice } \\
\text { characteristics and } \\
\text { satisfaction items } \\
\text { Free-text } \\
\text { interprofessional practice } \\
\text { items }^{\star}\end{array}$ & $\begin{array}{l}\text { Two years } \\
\text { postgraduation }\end{array}$ \\
\hline Survey 5 & $\begin{array}{l}\text { ATHCTS } \\
\text { TSS } \\
\text { Clinical practice } \\
\text { characteristics and } \\
\text { satisfaction items } \\
\text { Free-text } \\
\text { interprofessional practice } \\
\text { items }^{*}\end{array}$ & $\begin{array}{l}\text { Three years } \\
\text { postgraduation }\end{array}$ \\
\hline
\end{tabular}

*Items only completed by participants who attended the TIPE. ATHCTS, Attitudes Towards Health Care Teams Scale; TIPE, Tairāwhiti Interprofessional Education Programme; TSS, Team Skills Scale.

1. prior to the students' final year of preregistration training (survey 1; baseline);

2. at the end of students' final year of preregistration training (survey 2);

3. twelve months' postcourse completion (survey 3);

4. twenty-fourmonths' postcourse completion (survey 4);

5. thirty-six months' postcourse completion (survey 5 ).

Cohort 1 data will be collected between October 2014 and October 2018. Cohort 2 data will be collected between February 2016 and October 2019 (figure 3).

Cohort 1 survey 1 baseline data were collected by paper-based surveys and entered into an Access database (Microsoft, Redmond, Washington, USA). Cohort 2 baseline data and all subsequent data for both cohorts are collected through a web-based survey (IBM Data Collection; IBM, Armonk, New York, USA) administered by an independent research company. A lot of work went into 


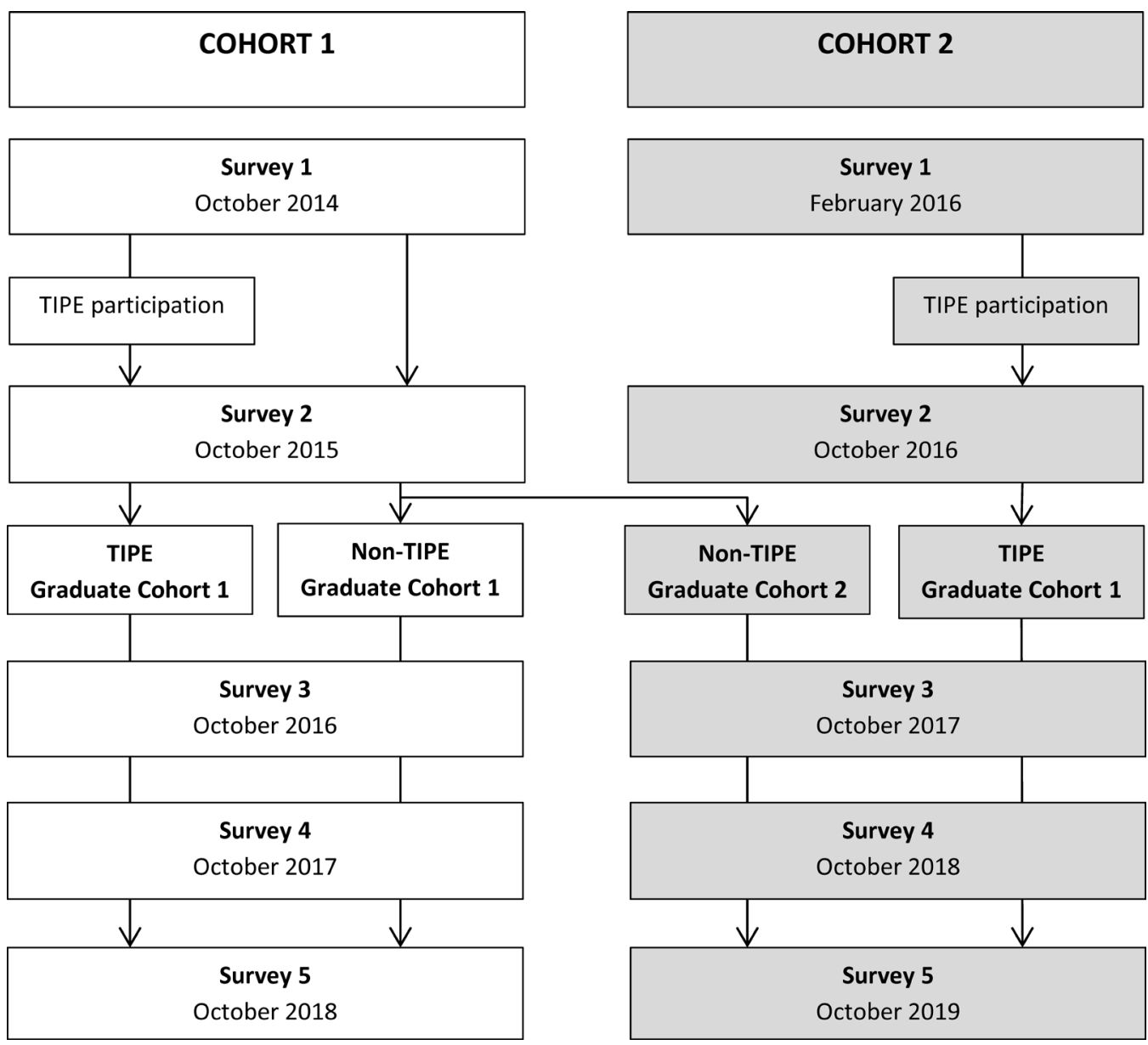

Figure 3 Overview of intervention exposure and survey dates for graduate cohorts 1 and 2. TIPE, Tairāwhiti Interprofessional Education Programme.

developing attractive, easy to use and electronic device-responsive surveys.

Students who do not graduate as expected-for example, those studying part time, deferring studies or failing to meet course requirements-will be accommodated as far as possible and appropriate within the study by completing later surveys at different time points. Participants who complete registration requirements before July of the following year will be included with their original cohort. Participants recruited as part of cohort 1 who complete registration requirements between July 2016 and June 2017 will be moved to graduate cohort 2. Participants who meet registration requirements after July 2017 will be removed from the study; it is not feasible to extend the study for 12 months to accommodate these few initial participants.

Survey 2 and survey 3 data collection instruments and methods were piloted with a group of nurses who graduated 6 months ahead of cohort 1 participants. This enabled the refinement of item wording and data collection processes. Data from these pilot participants will not be included in analyses. Surveys 4 and 5 repeat items from survey 3 and were not piloted.

Participants who complete surveys are entered in prize draws for each survey round with an additional prize draw for those who complete all five surveys. Postregistration respondents are also able to download a participation certificate which they may add to their Continuing Professional Development record.

\section{Participant retention}

Participant loss and non-response over time is a key challenge for longitudinal studies. The Longitudinal Interprofessional (LIP) study will employ a variety of techniques to minimise loss-to-follow-up. A wide range of contact details were collected at baseline (including mobile telephone number, email address and postal address as well as alternatives for each of these). Participants also have access to a web portal where they can update contact details and they are asked to confirm or update contact details as the first item within each survey. In addition, an interactive website which includes a discussion forum is maintained, and participants will receive communication from the study four times per annum using their choice of email or mobile phone text messaging; these communications will include endorsement from leaders in each discipline and from the three contributing educational institutions. Surveys 2 and 3 will be piloted with a separate cohort of health students/professionals to identify potential issues which could affect response rates. During survey rounds, 
responses will be encouraged by entering respondents in prize draws and providing participation certificates/ Continuing Professional Development points for survey completion. Non-respondents during each survey round will receive email, text message, telephone and/or postal follow-up.

\section{Analysis}

Quantitative aspect of questionnaire

Baseline characteristics (demographics, ATHCTS and TSS) will be compared for: the 2015 and 2016 TIPE students, the TIPE and non-TIPE students and the different disciplines. ATHCTS and TSS scores will be compared with t-tests/analysis of variance. Demographic items will be compared with Wilcoxon rank-sum tests/ Kruskal-Wallis tests or ${ }^{2}$ tests. The TIPE and non-TIPE comparisons will also be adjusted for discipline with linear regression, linear regression on ranks or logistic regression.

ATHCTS and TSS scores will be compared between Surveys 1 and 2 with paired t-tests for TIPE and non-TIPE students.

Mean ATHCTS and TSS scores will be calculated at each time point for cohort 1 , cohort 2 and combined TIPE, non-TIPE students and each disciplinary group. Mixed-model analysis of covariance will compare scores, adjusted for discipline, baseline demographics, ATHCTS and TSS, with terms for whether graduates participated in the TIPE programme, time of survey, the interaction of TIPE programme and time, and random terms for individual student.

Job and career satisfaction between those who did and did not participate in the TIPE programme will be compared with mixed-model analysis of variance with terms for discipline, TIPE programme and time of survey, the interaction of TIPE programme and time and random terms for student.

Professional setting and location data will be compared between those who participated in the TIPE programme and those that did not with mixed-model logistic regression with terms for discipline, TIPE programme, time, the interaction of TIPE programme and time, and random terms for student.

Associations between satisfaction data and discipline, professional setting and location will also be examined with additional terms added to the models.

The impact of loss-to-follow-up and missing data will be investigated with multiple imputation. The imputation model will include all the variables in the analysis model and demographic variables related to loss-to-follow-up or variables being missing.

\section{Qualitative aspect of questionnaire}

Data collected as free-text comments and question responses will be analysed using principles of template analysis. Template analysis is well suited to analysing responses to open-ended written questions, using the pre-prepared questions as a starting point, but also allowing for coding to be changed in response to the data as required. It provides a systematic way of approaching the data, that is particularly useful when a research team is undertaking qualitative analysis together, and yet is flexible enough to allow in-depth analysis. ${ }^{35}$

An initial coding template matrix will be developed from a subset of data, but modified as necessary, as more data are considered and data analysis proceeds. Responses will then be mapped into themes and subthemes. According to principles of template analysis, modification will be repeated at intervals until all or very nearly all the data can be satisfactorily mapped to the themes and subthemes. Ultimately, the coding structure and resultant themes will be highly relevant to the research questions, within disciplinary groups, within those who did and did not participate in the TIPE programme and across the entire cohort. Analysis will explore notable variation and similarities over the course of the study.

\section{Sample size}

No formal sample size calculation was undertaken because cohort 1 included all students eligible to attend the TIPE programme in 2015. It was obvious that the small portion of students from this cohort who attended TIPE would limit power to find any differences that existed between these students' outcomes and that of their peers. It was not feasible to recruit an additional complete-year cohort; however, an additional cohort of TIPE participants was recruited from the subsequent year group to increase the size of the TIPE sample. The investigators are not aware of any major changes in curriculum between 2015 and 2016 which would influence the comparability of the cohort 1 participants to those from cohort 2.

\section{Strengths and limitations}

This research will be one of very few longitudinal studies of IPE and of early career trajectories for newly qualified health professionals. ${ }^{19}$ The study design will allow comparisons to be made between students who did and did not participate in an IPE immersion programme with regard to attitudes to interprofessional teams and self-assessed ability to function within a team, workplace location and vocation and career satisfaction. However, it is recognised that the instruments available to measure the IPE outcomes have limitations which may contribute to failures to detect change. ${ }^{36}$ Typically, new graduates are encouraged to work in secondary care or must do so as part of their postregistration training, this may result in insufficient power to conduct meaningful comparisons in terms of graduates working in rural environments or primary healthcare. The addition of cohort 2 is designed to increase this power. There may also be a number of important confounding influences on career choices such as familial requirements, job availability or training programme requirements. These will be explored through qualitative free-text responses that ask participants to explain their choices. Follow-up 3years postregistration may not be sufficient to capture where participants settle 
in terms of career choice, practice setting and geographical location. Participant consent and funding will be sought to continue with data collection beyond 3 years.

Allocation to the TIPE programme is not random and varies between disciplines according to requirements for final year study. There are two main steps in selecting students to attend the TIPE programme. The first step involves planning the dates of the five training block dates; this is done in July the previous year. At this point, the disciplinary composition and quantity of students from each discipline for each block is decided depending on matches against disciplinary timetables, available accommodation and TIPE programme staff availability. Following these decisions, each discipline selects the students to fill the allocated spots. There are differences in how each discipline manages this process but the majority ask the students to apply in writing or rank their placement options. Disciplines then consider both the applications and whether the TIPE programme fits within each student's timetable. Where TIPE places are not filled by this process, disciplines choose students who are available but have not necessarily have applied to go to the TIPE programme. Consequently, many students (but not all) deliberately choose to attend the programme, and this may be related to their interest in interprofessional practice, rural health and/or hauora Māori. Baseline attitudes to collaborative teamwork will be used to adjust analysis of covariance to control for TIPE participants being more positive about interprofessional practice at baseline.

Although there will be a comparator group for the primary analyses related to interprofessional attitudes and self-assessed competencies, there will not be for freetext items answered by TIPE participants such as experiences of working as part on an interprofessional team, the influence of preregistration training on their preparedness to work within interprofessional teams or the influence of the TIPE programme on their career choices. Analysis of these qualitative data will draw on the methodology of a longitudinal case study with assessment at key time points where important changes are anticipated to occur. ${ }^{37}$ Multiple data sources (self-reported attitudes and abilities as well as qualitative data) will contribute to the evaluation and help to explain results. ${ }^{37}$

The TIPE programme represents just 5 weeks out of 3 to 6 years of each discipline's training, and a small number of students may have been exposed to other (although considerably less intensive and/or informal) interprofessional experiences during their preregistration training. Although exposure to the TIPE programme will be the key difference between graduates who did and did not participate, there may be other factors which influence graduates' attitudes and career choices and may confound analyses. Graduates' reports of important influences will also need to be interpreted in light of recall and desirability biases.

There are a number of challenges associated with a longitudinal study of health professional students and graduates. These include students not graduating with their recruitment year groups; the study covering a period of great change for a group of predominantly young and mobile health professionals when they move from training to employment during which places and country of residence, email addresses and other contact details change. These factors may reduce the ability of the study to follow these participants and achieve high response rates.

\section{ETHICS AND DISSEMINATION}

Results will be shared with study participants through the study website and the host department's website. Findings will be disseminated through peer-reviewed publications, national and international conferences and reports to the university and health policy stakeholders. In particular, this will include Health Workforce New Zealand (a subunit of the country's Ministry of Health) who provided funding to initiate the TIPE programme in 2012.

It is anticipated that this study will provide new information regarding the development of interprofessional attitudes and skills during the final year of preregistration training and first 3 years of professional practice, as well as the influence of an IPE immersion programme on these.

Very little is currently known about the career trajectories of newly qualified health professionals. The current study will build on the few existing studies by providing an opportunity to compare between a diverse range of the key disciplines in healthcare and also allowing comparisons to be made between those who participate in the rurally based TIPE programme and others within their year group. Although in New Zealand there is ongoing data collection for some discipline-specific databases which capture aspects of health professional early career trajectories, these are currently limited to medicine (Medical Schools Outcomes Database) ${ }^{38}$ and physiotherapy (Physiotherapy New Graduate Survey). Neither investigates the nature of any collaborative practice, and there is no other comparable data collection for other health discipline graduates. Key goals of the TIPE programme are to increase rural and primary care career choices. Data gathered by this study may indicate if it is achieving these objectives.

Analysis of qualitative data captured from those who participated in the TIPE programme will contribute to understanding of recently graduated professionals' participation in interprofessional teams and the influence of preregistration IPE on career trajectories. These analyses will help inform future initiatives to increase selection of careers within rural or primary care environments and inform preregistration training development which prepares new graduates to effectively function with interprofessional teams.

Acknowledgements The research team gratefully acknowledge the contribution of Katrina Magill, Pip Sutton and Sarah Buchanan from Research New Zealand, the TIPE Governance Group and Education Operations Group, and staff at each participating school for facilitating the study.

Collaborators LIP Study Group: Louise Beckingsale, Kaye Cheetham, Jennifer Roberts, Ruth Crawford, Sue Floyd, Lyndie Foster Page, Janice Handley, Jackie 
Herkt, Lisa Kuperus, Patrick McHugh, Alison Meldrum, Margot Skinner, Rose Schwass, Julie Weaver and James Windle.

Contributors BD, PG, LG, EM, CW and SP contributed to the conception and design of the study. BD, GP and SP developed the analysis plan. BD is the guarantor and drafted the initial protocol. BD, MB and GP analysed and presented the data for participant flow diagrams. BD, MB, PG, LG, EM, GP, CW and SP revised the protocol critically for important intellectual content and read and approved the final version of the manuscript to be published.

Funding This study is supported by a grant from the University of Otago Division of Health Sciences.

Disclaimer The funder had no role in the study design, collection, analysis and interpretation of the data; writing of the protocol, or in the decision to submit the paper for publication.

Competing interests None declared.

Patient consent Not required.

Ethics approval This study has received approval from the University of Otago Ethics Committee (D13/019).

Provenance and peer review Not commissioned; externally peer reviewed.

Open Access This is an Open Access article distributed in accordance with the Creative Commons Attribution Non Commercial (CC BY-NC 4.0) license, which permits others to distribute, remix, adapt, build upon this work non-commercially, and license their derivative works on different terms, provided the original work is properly cited and the use is non-commercial. See: http://creativecommons.org/ licenses/by-nc/4.0/

(C) Article author(s) (or their employer(s) unless otherwise stated in the text of the article) 2018. All rights reserved. No commercial use is permitted unless otherwise expressly granted.

\section{REFERENCES}

1. Lapkin S, Levett-Jones T, Gilligan C. A systematic review of the effectiveness of interprofessional education in health professional programs. Nurse Educ Today 2013;33:90-102.

2. Oandasan I, Barker K, Barker C, et al. Teamwork in Healthcare: promoting effective teamwork in health care in Canada. Ottawa: Canadian Health Services Research Foundation, 2006.

3. Thistlethwaite J. Interprofessional education: a review of context, learning and the research agenda. Med Educ 2012;46:58-70.

4. Wagner EH. Meeting the needs of chronically ill people. BMJ 2001;323:945-6.

5. Hall P. Interprofessional teamwork: professional cultures as barriers. J Interprof Care 2005;19:188-96.

6. Oandasan I, Reeves S. Key elements of interprofessional education. part 2: factors, processes and outcomes. J Interprof Care 2005;19:39-48.

7. Institute of Medicine. Measuring the impact of interprofessional education on collaborative practice and patient outcomes. Washington: DC: IOM, 2015.

8. World Health Organisation. Framework for action on interprofessional education and collaborative practice. Geneva: World Health Organisation, 2010.

9. Nelson S, White CF, Hodges BD, et al. Interprofessional team training at the prelicensure level: a review of the literature. Acad Med 2017;92:709-16.

10. Darlow B, Coleman K, McKinlay E, et al. The positive impact of interprofessional education: a controlled trial to evaluate a programme for health professional students. BMC Med Educ 2015;15:98.

11. Hammick M, Freeth D, Koppel I, et al. A best evidence systematic review of interprofessional education: BEME guide no. 9. Med Teach 2007;29:735-51.

12. Chakraborti C, Boonyasai RT, Wright SM, et al. A systematic review of teamwork training interventions in medical student and resident education. J Gen Intern Med 2008;23:846-53.

13. Reeves S, Goldman J, Burton A, et al. Synthesis of systematic review evidence of interprofessional education. J Allied Health 2010;39:198-203.
14. Reeves S, Perrier L, Goldman J, et al. Interprofessional education: effects on professional practice and healthcare outcomes (update). Cochrane Database Syst Rev 2013;3:CD002213.

15. Cox M, Cuff P, Brandt B, et al. Measuring the impact of interprofessional education on collaborative practice and patient outcomes. J Interprof Care 2016;30:1-3.

16. Coster S, Norman I, Murrells T, et al. Interprofessional attitudes amongst undergraduate students in the health professions: a longitudinal questionnaire survey. Int J Nurs Stud 2008;45:1667-81.

17. Curran VR, Sharpe D, Flynn K, et al. A longitudinal study of the effect of an interprofessional education curriculum on student satisfaction and attitudes towards interprofessional teamwork and education. J Interprof Care 2010;24:41-52.

18. McFadyen AK, Webster VS, Maclaren WM, et al. Interprofessional attitudes and perceptions: results from a longitudinal controlled trial of pre-registration health and social care students in Scotland. $J$ Interprof Care 2010;24:549-64.

19. Pollard KC, Miers ME. From students to professionals: results of a longitudinal study of attitudes to pre-qualifying collaborative learning and working in health and social care in the United Kingdom. $J$ Interprof Care 2008;22:399-416.

20. Wilhelmsson M, Pelling S, Ludvigsson J, et al. Twenty years experiences of interprofessional education in linkoping-groundbreaking and sustainable. J Interprof Care 2009;23:121-33.

21. Minister of Health. New Zealand health strategy: future direction. Wellington: Ministry of Health, 2016.

22. Pullon S, McKinlay E, Gallagher P, et al. In: Bin Abdulrahman $\mathrm{K}$, Mennin S, Harden R, eds. Interprofessional education in a rural clinical setting: a quick start innovation for final year health professional students-case study. New York: Routledge International Handbook of Medical Education, 2015:197-9.

23. Pullon SS, Wilson C, Gallagher P, et al. Transition to practice: can rural interprofessional education make a difference? A cohort study. BMC Med Educ 2016;16:154.

24. McKinlay E, Gallagher P, Wilson C, et al. Social learning, shared accommodation and interprofessional education: i think those conversations that you have at the dinner table. J Interprof Educ Pract 2016;5:1-6.

25. Heinemann GD, Schmitt MH, Farrell MP, et al. Development of an attitudes toward health care teams scale. Eval Health Prof 1999;22:123-42.

26. Curran VR, Sharpe D, Forristall J. Attitudes of health sciences faculty members towards interprofessional teamwork and education. Med Educ 2007;41:892-6.

27. Hayashi T, Shinozaki H, Makino $\mathrm{T}$, et al. Changes in attitudes toward interprofessional health care teams and education in the first- and third-year undergraduate students. J Interprof Care 2012;26:100-7.

28. Curran VR, Sharpe D, Forristall J, et al. Attitudes of health sciences students towards interprofessional teamwork and education. Learning in Health and Social Care 2008;7:146-56.

29. Hepburn K, Tsukuda RA, Fasser C, et al. Team skills scale. In: Heinemann GD, Zeiss AM, eds. Team performance in health care: assessment and development. New York: Kluwer Academic/Plenum Publishers, 2002

30. Curran V, Heath $\mathrm{O}$, Adey T, et al. An approach to integrating interprofessional education in collaborative mental health care. Acad Psychiatry 2012;36:91-5.

31. Fulmer T, Hyer K, Flaherty E, et al. Geriatric interdisciplinary team training program: evaluation results. J Aging Health 2005;17:443-70.

32. Dolbier CL, Webster JA, McCalister KT, et al. Reliability and validity of a single-item measure of job satisfaction. Am J Health Promot 2005;19:194-8.

33. Highhouse S, Becker AS. Facet measures and global job satisfaction J Bus Psychol 1993;8:117-27.

34. Landon BE, Reschovsky J, Blumenthal D. Changes in career satisfaction among primary care and specialist physicians, $1997-$ 2001. JAMA 2003:289:442-9.

35. Brooks J, McCluskey S, Turley E, et al. The utility of template analysis in qualitative psychology research. Qual Res Psycho 2015;12:202-22

36. Oates M, Davidson M. A critical appraisal of instruments to measure outcomes of interprofessional education. Med Educ 2015;49:386-98.

37. Yin R. Case study research design and methods. 5th edn. Thousand Oaks, CA: Sage Publications, 2014.

38. Kaur B, Carberry A, Hogan N, et al. The medical schools outcomes database project: Australian medical student characteristics. BMC Med Educ 2014;14:180. 